\title{
IFAS Assessment of the Status of Non-Native Plants in Florida's Natural Areas ${ }^{1}$
}

Authors:

Alison M. Fox, Doria R. Gordon, Joan A. Dusky, Linda Tyson, and Randall K. Stocker²

\section{Footnotes:}

1. This document is SS-AGR-225, one of a series of the Agronomy Department, Florida Cooperative Extension Service, Institute of Food and Agricultural Sciences, University of Florida. Revised publication date: July 2004.

In IFAS Extension publications this document should be cited as:

"IFAS Assessment, 2004. IFAS assessment of non-native plants in Florida's natural areas. Cited from the World Wide Web http://agronomy.ifas.ufl.edu/IFASassessmt.HTML"

2. Alison M. Fox, Associate Professor, Agronomy Department, University of Florida, Gainesville, FL; Doria R. Gordon, State Ecologist, The Nature Conservancy and Courtesy Professor, Department of Botany, University of Florida, Gainesville, FL; Joan A. Dusky, Professor and Associate Dean for Extension, University of Florida, Gainesville, FL; Linda Tyson, Associate Professor, Santa Fe Community College, Gainesville, FL; and Randall K. Stocker, Director, Research and Outreach/Extension, UF School of Natural Resources and Environment, and Professor, Agronomy Department, University of Florida, Gainesville, FL. 


\section{Purpose}

The purpose of this assessment is to provide a well-defined mechanism by which all IFAS Extension publications can conform in their description and categorization of non-native plants that are invading natural areas in Florida. Conclusions derived from this assessment are intended to prevent, or reduce, the likelihood of further invasion of natural areas by non-native plants. Development of a common basis for decisions will increase consistency and understanding of recommendations made by IFAS personnel concerning invasive, non-native plants. This assessment has rigorous requirements for documentation to support all relevant evidence and this reinforces the transparency and credibility of the process. It is intended that this assessment will be useful in developing priorities for research and management efforts, and that it can be adapted for use in other states (i.e., with appropriate zonal definitions).

This assessment is not a predictive instrument but is intended ONLY for plant species or cultivars that currently occur within Florida. It is not intended to provide evaluations of species that have not yet been introduced to the State. Such species would require a separate predictive instrument, still to be developed.

It is likely that less than $1 \%$ of all non-native plant species in the State will be identified as showing ecological impacts in natural areas (for definitions of terms as used in this document see Glossary, pages 25 - 27). This assessment is designed to identify those non-native species that are invasive in areas of Florida with designated management objectives that include the conservation of native biodiversity. While a range of activities may be conducted in these areas, those activities are designed to be compatible with the conservation objective. State and local governments and some private landowners, for example, manage natural areas both for recreation, grazing, forestry or other harvest values and for conservation values. These areas would be included in the assessment of invasion, with clearly identifiable edges disregarded. Species that invade only adjacent to roads, trails, fire lanes, recent dredge spoil, expanses of bare soil, etc. should not be identified as invaders with impacts in natural areas because their persistence and spread is only in these anthropogenically disturbed zones in the natural area. Species that spread along these artificially disturbed zones but disperse over 10 yards into more intact natural areas would be required to be evaluated by this assessment.

It is also recognized that some anthropogenically disturbed areas can provide habitat for species of special concern; thus in considering broad conservation objectives, it may still be necessary to review non-native species that have not been identified in this assessment as invading natural areas. For example, where Threatened or Endangered species are now predominantly found and maintained in anthropogenically disturbed areas, it is possible that these could be impacted by non-native species that are described by this assessment as "Not considered a problem species at this time". Such cases should be referred to the Florida Endangered Plant Advisory Council (Secretary: Danny Phelps, FL Department of Agriculture and Consumer Services, Division of Plant Industry, Tel: 352-372-3505.) 


\section{Objective}

The objective of this assessment is to summarize relevant ecological, management and economic value information on species. The intention is to provide a level of information divided into relevant categories that is greater and more functional than that indicated by simple presence or absence from an "Invasive Species List." This information is summarized by four indices, Ecological Impacts, Potential for Expansion, Difficulty of Management and Economic Value. Where possible, predictive items have been avoided. Most questions have been reduced to simple two (or occasionally three) choice answers. This assessment does not address economic impacts other than management costs and economic value. Such data (lost revenue, management costs other than control in natural areas, etc.) should be included in any detailed risk-benefit analyses of the current, or future, infestations of a species.

This assessment will be generally applied at the species level. It will only be applied independently to cultivars (varieties) or hybrids of a species if there are differences among those cultivars that would result in different responses to the assessment categories. In all cases, the location of a voucher specimen must be identified. (Throughout the assessment, reference to the species under consideration could also refer to a cultivar or hybrid.) For each species, separate conclusions are derived from the assessment for each of three latitudinal zones within Florida.

The conclusions include a period after which the species must be reassessed (either 10 or 2 years). However, any species may be reassessed whenever additional relevant information is obtained that changes the results of the assessment. Thus, conclusions might change at any time.

\section{Use of This Assessment Within IFAS}

Species will be assessed by trained personnel under the supervision of the IFAS Invasive Plant Working Group. The results of each assessment will be made available to all IFAS state and county Extension faculty (hereafter "IFAS faculty") through the web site for the Center for Aquatic and Invasive Plants (http://plants.ifas.ufl.edu/) with links to IFAS Extension web sites. IFAS faculty dealing with non-native plants species in Florida are expected to be familiar with this assessment.

This assessment will place non-native plant species in categories (called "index categories"). These categories describe varying degrees of "Ecological Impacts", "Potential for Expansion", "Management Difficulty", and "Economic Value". Any IFAS Extension publications or newsletters, including those developed by county faculty, that refer to specific non-native plants in relation to the topics covered by this assessment (e.g., invasiveness, ecology, distribution, management, use, recommendations, and value) are required to include a reference to this assessment, and to use the terms and conclusions from the assessment when describing features of these plants. For example, “...shows high ecological impacts such as causing long-term alterations in ecosystem processes and... This species is considered invasive and not recommended by IFAS faculty (IFAS Assessment, 2004). ”

This assessment uses the index categories to define "conclusions" about the assessed species, including conclusions such as "Invasive and not recommended by IFAS faculty". Faculty making planting recommendations may choose to include "...not recommended..." plants in their publications, if the conclusions from the assessment are clearly presented. In some 
situations, it may provide better public education to include a "...not recommended..." plant with the reasons it should be avoided, instead of leaving the species out of the publication entirely.

This assessment will be revised periodically, and content and conclusions may change substantially. IFAS Extension publications must conform to the assessment protocol in place at the time of that publication's final drafting, and must be made to conform to the current assessment protocol when that publication is revised. The IFAS Invasive Plants Working Group will continue to review all Extension pre-publications related to invasive plants, and will determine if the direction and intent of these provisions are being followed.

\section{Structure of the Assessment}

The assessment is divided into five main sections, one for determining where the plant is invading and the others corresponding to four indices of Ecological Impacts, Potential for Expansion, Difficulty of Management, and Economic Value. These sections are designated by Roman Numerals $(I-V)$. Assessment questions within these sections are located in shadowed boxes which have sub-section letters (I-a, I-b, etc.). Separate questions within a box have their own number which is in either arabic numerals, for questions that influence the order of progression through the assessment (e.g., I-a 1., I-a 2.), or diminutive Roman numerals, for statements to which scores are assigned (e.g., II-a i, II-a ii). Three further sections that directly lead to conclusions have letters $A-C$ and are found at the end of the assessment.

\section{Instructions}

For each species, cultivar or hybrid, complete a new copy of the Response Form and indicate the location of a voucher specimen. Where appropriate, references, personal communications and observations should be noted. This will provide important documentation of data used in the assessment for future reviews.

It is important to establish that anyone whose input is sought is qualified to comment on a particular species (i.e., they are confident of its identification in the field, and for the zone(s) that they can comment on, they are familiar with the types of habitat in which this species is likely to occur.)

If at any stage in this assessment you realize that you do not have sufficient information to make a decision that will determine where to proceed next in the assessment,

\section{STOP!}

Indicate on the front of the response form which answer is unknown and try to recommend who might be able to provide the answer. 
Certain species, currently existing in Florida or not yet known there, are prohibited by federal or state laws. IFAS faculty will not recommend such species and should note their prohibited status when discussing them. Relevant lists of such species can be found at:

USDA / APHIS - Federal noxious weed list

http://www.aphis.usda.gov/ppq/permits/noxiousweed list.html

Dept. Agric. and Consumer Services - Noxious weed list

http://www.doacs.state.fl.us/pi/noxious-list.htm

Dept. Environmental Protection - Prohibited plant list

http://www.dep.state.fl.us/lands/invaspec/index.htm

\section{Automatic Exemption From the Assessment}

Is this species listed on any federal or state noxious, or prohibited plant lists?

If YES then do not proceed with assessment but indicate a conclusion of:

Prohibited on the front of the response form

If NO then Go to Section I (page 6) 


\section{Section I Invasion Status}

The purpose of this section is to separate species that do not appear to invade natural habitats from those that do. For this assessment, a species is defined as invading if it forms selfsustaining and expanding populations within a natural plant community with which it had not previously been associated (cf. "invasive" in Vitousek et al. 1995). This definition does not specify that the species must cause some defined impact (as is required by some definitions) because this will be addressed separately.

\section{I-a Current Invasion in Florida}

1. Does this species occur in any natural areas of Florida? Provide distributional evidence (as defined in the glossary).

\section{If NO then Go to Section A (page 21) If YES then Go to question I-a 2.}

2. Does it ONLY occur in natural areas of Florida because it has persisted from its previous cultivation in that location (e.g., in abandoned farmland or homesteads)?

If YES then Go to Section A (page 21) If NO then Go to Section I-b (Below)

\section{I-b Invasion Status in Three Zones of Florida}

Some species may be invading all parts of the state, while others can invade one zone (e.g., subtropical south Florida) but may not be able to invade or survive in other zones. A few species may be found currently in only one zone but have the potential to continue to spread to others. Having determined the invasion status of the species on a State-wide basis in Section I-a, indices for Ecological Impacts (Section II) and Potential for Expansion (Section III) are determined separately for each of three latitudinal zones so that conclusions can be proposed that account for varying latitudinal and environmental patterns of invasion. In zones where a species is not currently invading, only Potential for Expansion will be evaluated (Section III).

The State of Florida has been divided into three phytogeographic regions based on an estimated chill accumulation chart. We have modified that map to separate zones at the northern border of counties that lie along the 420 chill unit accumulation (north) and 110 chill unit accumulation (south) boundaries. These zone are similar to those defined by USDA Hardiness Zones 8, 9 and 10/11 (http://www.usna.usda.gov/Hardzone/index.html). 


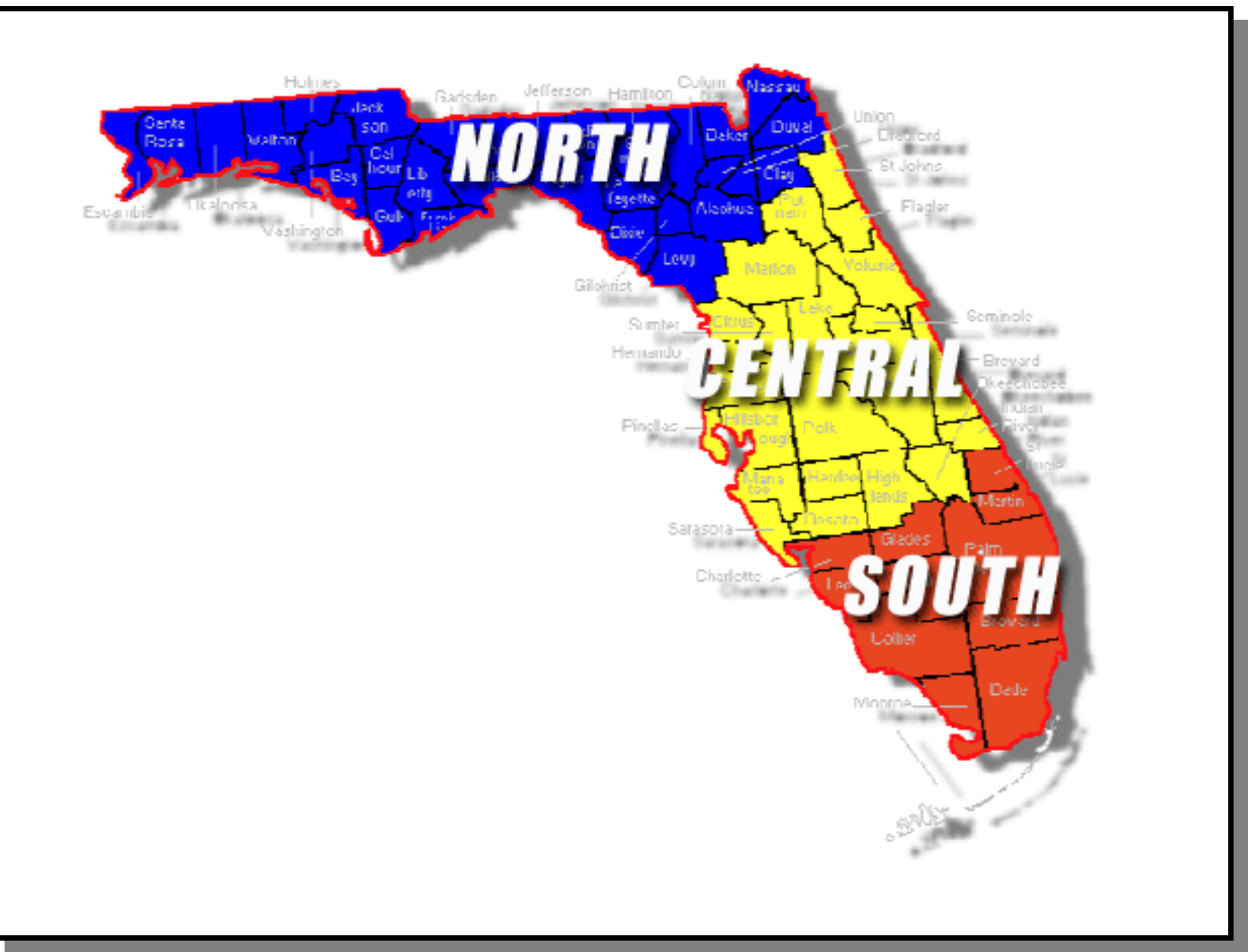

While no areas of Florida are wholly undisturbed by human activity (e.g., air pollution, trails, fragmentation), this assessment is confined to species that invade natural areas in which that anthropogenic disturbance is minimal, or species that may persist in natural communities once anthropogenic disturbances have been largely removed.

Natural systems are dynamic because of a number of natural disturbances at various scales (e.g., gopher mounds, treefalls, fire, tides, flooding, hurricanes, etc.). Altering the frequency and scales of those natural disturbances presents as much of a perturbation to the system, that may allow establishment of new species, as does introducing a completely new anthropogenic disturbance (e.g., trampling, grazing animals, soil movement, drainage, pollution, etc.). If a species invades only when disturbance regimes other than those under which the community evolved occur, and is not known to persist when the natural regime is reestablished, this species will not have critical impacts in natural areas being managed to restore or maintain natural processes. For example, skunk vine (Paederia foetida) can invade sandhills that have been fire suppressed, but it is quickly killed and is maintained at low levels when fire is restored to that system. (Note: skunk vine can also invade undisturbed areas and non fire-adapted habitats, so it would still be categorized as invading in Section I-b.)

A species that can establish and persist when natural disturbance regimes exist may pose a serious threat to natural systems. Non-native species that do not require an anthropogenic or other novel disturbance for establishment are pre-adapted to Florida's natural systems and may out-compete native species also adapted to those systems. 
I-b Invasion Status in Three Zones of Florida

Determine responses to the following questions for one zone (north, central, south) at a time.

Distributional evidence (as defined in the glossary) for invasion (forming self-sustaining and expanding populations within a plant community with which it had not previously been associated) must be provided for YES answers to questions $1-3$.

1. Does this species exist in any areas outside its current, or former, location of cultivation in this zone?

If YES then Go to question I-b 2. If NO then Go to question I-b 4.

2. Is this species invading in this zone ONLY when natural disturbance regime and scale have been altered? (e.g., where frequency, extent, or severity of fires have been reduced by human activity.)

If YES then Go to question I-b 3.

If NO then Go to Section II (page 9)

3. Has this species ever been known to persist, following colonization, when the natural regime is resumed and the natural flora/communities recover? (E.g., is not an early successional species that only temporarily invades disturbed areas.)

If YES (or unknown) then Go to Section II (page 9) If NO (known not to persist) then Go to question I-b 4.

4. Are there other zones in which this species: EITHER has invaded when there was no alteration of the natural disturbance regime, OR has persisted following the restoration of natural disturbance regimes?

If YES then Impact score $=\mathbf{0}$ for this zone and Go to Section III-b (page 13) If NO then Go to Section A (page 21) 
Section II Ecological Impacts of Invasion

Impact Index

The purpose of this section is to evaluate the severity of ecological impacts caused by an invasion. This evaluation is made without regard to the actual size or age of an invasion, and is independent of any assessment of value or cost of the species. Because we are defining invasion without a requirement for impact (see Section I), it is possible in this assessment for a species to have been identified as invading natural habitats but to score low or zero on the Ecological Impact scale (e.g. remains at a low density in an existing stratum of vegetation). Other species may show multiple impacts, which will result in a high score.

In the first part of this section the worst known impacts of a species are assessed regardless of whether these worst impacts are widespread.

\section{II-a Known Impacts at WORST SITE(S) (without, or before, any control effort)}

Add up points for ALL impact statements (i through vi) that are true at the worst affected

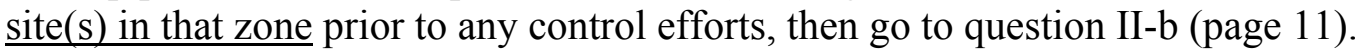

Documentation of evidence (as defined in the glossary) must include specific locations of observed impacts. Scientific names of impacted species (e.g., Species of Special Concern, Threatened or Endangered, or native species with which hybridization occurs) must be included. [If insufficient evidence of ecological impacts is available for zone X, continue with the assessment to develop conclusions for the other zones. If directions on page 20 cause Section $C$ to be used in zone X and the answers to questions $C 1$ and $C 2$ are "yes", then the lack of sufficient evidence of ecological impacts does not preclude completion of the assessment - see page 23. Under such circumstances, the Ecological Impact category for zone $X$ is based on the sum of scores only for impact statements that have been confirmed in the literature or by two experts. If Section $C$ is not used or an answer to C1 or C2 is "no", then sufficient evidence needs to be gathered in all zones to complete the assessment for a particular species.]

If there is no evidence of an impact, then assign 0 points unless the impact is considered very likely (e.g., fixes $\mathrm{N}_{2}$ in low nutrient soil which can change the flora) OR the impact (except vi) has been demonstrated in similar habitats in other zones or outside the state. In these cases assign 0.5 points.

i) Causes long-term alterations in ecosystem processes (see glossary)

Points influencing multiple species (e.g., changes fire regime; increases shoreline sedimentation). 
ii) Has negatively impacted Federal- or Florida-listed Species of Special

Concern or Threatened or Endangered plants or animals.

Choose one of the following:

Displacement, death or hybridization has occurred (document specific locations of Species of Special Concern, Threatened or Endangered species).

Impacts are considered likely because Federal- or Florida-listed Species of Special Concern, Threatened or Endangered species and the invading species closely co-habit (e.g., compete for light).

iii) Displaces or precludes native vegetation (affecting mortality and/or recruitment) by achieving populations in the zone that have at least $50 \%$ coverage of this species (as defined in the glossary) in the affected stratum that meet any of the following criteria:

a) collectively add up to at least 10 acres

b) are 5 populations of at least 0.25 acres each

c) are 5 populations that cover an entire localized community (e.g., sinkhole)

d) are 5 populations some of which are at least 0.25 acres and others of which cover entire localized communities.

iv) Changes community structure in ways other than vegetation displacement (e.g., alters wildlife abundance, adds a new stratum, or increases total stem density within a stratum by more than 5 -fold).

v) Hybridizes with native Florida plants or economically-important species.

vi) Covers over $15 \%$ of invaded stratum (but if 8 points were assigned for statement iii, do not assign points here). 
II-b Range of Communities in Which Species is Invading

Species that are capable of invading a wide range of communities are likely to have wide environmental tolerances and broader impacts than those species that are limited to a narrow range of communities. The following lists of community groups have been adapted from the Natural Community Groups defined by the Florida Natural Areas Inventory (FNAI and FDNR 1990).

These groups are described at: http://www.fnai.org/PDF/Natural Communities Guide.pdf

Terrestrial List

Xeric uplands - Sandhill, scrub, xeric hammock

Coastal uplands - Beach dune, coastal -berm, -grassland, -rock barren,-strand, maritime hammock, shell mound

Mesic uplands - Bluff, slope forest, upland -glade, -hardwood forest, -mixed forest, -pine forest

Rocklands - Pine rockland, rockland hammock, sinkhole

Mesic flatlands - Dry prairie, mesic flatwoods, prairie hammock, scrubby flatwoods

\section{Palustrine (wetland) / Aquatic List}

Wet flatlands - Hydric hammock, marl prairie, wet flatwoods, wet prairie

Seepage wetlands - Bay gall, seepage slope

Floodplain wetlands - Bottomland forest, floodplain - forest, -marsh, - swamp, freshwater tidal swamp, slough, strand swamp, swale

Basin wetlands - Basin -marsh, -swamp, bog, coastal interdunal swale, depression marsh, dome swamp

Lakes and rivers - All types of freshwater lakes, rivers and streams

Tidal wetlands - Marine and estuarine tidal marshes and swamps

\section{II-b Range of Community Groups in Which Species Has Impacts}

Is this species known to be invading at least four community groups OR does it occur in at least one community group of each of the terrestrial and palustrine/aquatic lists? (Within each zone, different community groups that are identified by various sources or experts are additive.)

If YES then Multiply total score from II-a by 1.5 then Go to Section II-c (below)

\section{II-c Proportion of Invaded Sites with Significant Impacts}

This section is intended to separate those species that do not cause serious impacts everywhere they occur but may do so only in very specific regions or environments. Such species may be acceptable for use in areas that are sufficiently distant from the specific communities that are impacted (Section B, page 22). It is important to estimate the proportion of sites where worstcase impacts have been recorded, relative to all sites within the zone where the plant has been invading.

\section{II-c Proportion of Invaded Sites with Significant Impacts}

Of the invaded sites, might any of the worst [statements i to $\mathrm{v}$ in Section II-a] impacts only occur under a few, identifiable, environmental conditions (i.e., conditions that occur in only 1 to $10 \%$ of the sites)? Documentation of evidence (as defined in the glossary) must be provided for a YES answer.

If NO OR no score on statements i to v in section II-a then Go to Section III (page 12) If YES then Go to Section B (page 22) 
Section III Potential for Expansion.

Potential Index

The purpose of this section is to provide a crude evaluation of whether there is high or low potential for this species to expand much further within Florida. This assessment is made independently for zones where the plant has invaded, and zones where it has not invaded. The assessment is made without regard to absolute areas of invasion or potential habitats. A high potential for expansion is indicated if there is a significant rate of spread of the species. This section is important for species that may have only recently invaded and for which little evidence of impacts may be available. It is also important for assessing the species' potential for expansion and causing impacts in zones which it is not currently invading.

\section{Question III-a ONLY For Zones Where Plant Has Invaded.}

III-a Known Rate of Invasion.

Doubling times and absolute areas can be difficult to quantify, so this section uses the concept of "discrete populations" to indicate the rate of habitat invasion. Discrete populations (populations separated by at least 1 mile - see glossary) are intended to be units that are easy to quantify and that probably indicate invading plant populations that arose by long-distance dispersal mechanisms. The rate at which new populations for a species are reported may be as much influenced by increased search-effort as actual expansion of plant coverage. However, this parameter is included because such change in reporting is typical of recently identified species, which are also most likely to have the greatest potential to expand beyond their current distribution.

\section{III-a Known Rate of Invasion.}

1. Was this species reported in more than two new discrete populations (at least 1 mile apart) in any 12 month period within the last 10 years? This should be based on populations only in natural areas and prior to management. Provide distributional evidence (as defined in the glossary).

If Unknown or NO then $\mathbf{P}=$ Low then Go to Section IV (page 15) If YES then $\mathbf{P}=$ High then Go to Section IV (page 15) 


\section{Question III-b ONLY For Zones Where Plant Has NOT Invaded.}

\section{III-b Potential for Invading Non-invaded Zones}

If a species is capable of surviving, reproducing, and dispersing in a zone then it is likely that its current absence from that zone is a function of its rate of expansion from the place where it was introduced to the State. Its potential for expansion once it reached a suitable new zone is likely to be high if its potential in currently invaded zones is high.

Documentation of evidence or provision of distributional evidence (as defined in the glossary) must be provided for a YES answer.

III-b $\quad$ Potential for Invading Non-invaded Zones

1. Would this species be able to survive, reproduce, and disperse in the climate of this zone? (E.g., based on comparisons of climate maps for Florida and worldwide locations where the species is known to occur, including invaded zones and/or native range.)

\section{If NO then $\mathbf{P}=$ Low and Go to Section IV (page 15) If YES then Go to question III-b 2.}

2. Do habitat-types suitable for the growth of this species occur in this zone? (E.g., based on species' known occurrence worldwide within invaded zones or native range.)

If NO then $\mathbf{P}=$ Low and Go to Section IV (page 15)

If YES then $\mathbf{P}$ for this non-invaded zone is equal to the $\mathbf{P}$ assigned to the nearest invaded zone (from Section III-a - if there is an option between two zones with $\mathbf{P}=$ High or Low, use High).

If an adjacent zone is invaded then Go to Section III-c (page 14) If the only adjacent zone is non-invaded then Go to Section IV (page 15) 
Question III-c

ONLY For Zones Where Plant Has NOT Invaded But Has The Potential To.

III-c Potential for Causing Ecological Impacts in Non-invaded Zones

The purpose of this section is to identify the likelihood of future ecological impacts in a zone that has not yet been invaded but which has the potential to be. If there is no reason to believe that the ecological impacts observed elsewhere in the State could not occur in this zone, then it is reasonable to adjust the ecological impact score to match that of an adjacent invaded zone, which will lead to similar conclusions for the two zones.

Documentation of evidence or provision of distributional evidence (as defined in the glossary) must be provided for a YES answer.

III-c Potential for Causing Ecological Impacts in Non-invaded Zones

1. For zones invaded by this species, identify all communities in which any ecological impacts identified in Section II-a occur. Do these communities occur in the uninvaded zone under consideration? (E.g., do the negatively impacted Federal- or Florida-listed Species of Special Concern, Threatened or Endangered species occur in this zone?) If no impacts were documented in any zones for this species, the response here is NO.

If NO then Go to Section IV (page 15)

If YES then revise the Ecological Impact Score for this zone from zero to match the Ecological Impact Score for the adjacent invaded zone (use highest value if there is an option; mark this revised score with brackets \{\} to show this score was derived from Section III-c) then Go to Section IV (page 15). 
Section IV Difficulty of Management

Management Index

It seems unlikely that management difficulty will vary significantly by zones so this section is assessed on the State-wide distribution of the species, NOT by zones. The intention of this section is to distinguish species for which management is especially difficult. For most statements, no particular methods of control are specified but responses should relate to the methods that are most likely to be used. Most of these statements relate to the availability of control methods, factors that influence the cost of control (over multiple generations, if relevant), or factors that indicate the increasing likelihood of unavoidable non-target damage. Management of species that become reproductive in a short period of time, or that produce many, long-lived and/or widely dispersed reproductive propagules, is more difficult because they will require more frequent, longer-lasting, and/or more widely ranging re-surveys and re-treatments.

Biological control can greatly reduce management costs, and several effective programs for biological control of non-native plants have been implemented in Florida. Because target plant species for biological control programs have, to date, been on Florida's Prohibited Aquatic Plants List (maintained by Florida Department of Environmental Protection) or Noxious Weeds List (maintained by Florida Department of Agriculture and Consumer Services), and because listed prohibited plants are automatically exempted from the assessment (page 5), neither the use nor the results of biological control are specifically addressed in this section.

This section is assessed on the State-wide distribution of the species, NOT by zones.

IV Factors That Increase the Difficulty of Management (As appropriate, statements should be assessed without, or before, any control effort)

Documentation of evidence (as defined in the glossary but agency reports can be substituted for original research) must be provided. Add up all points from statements that are true for this species then go to Section V (page 17). Assign 0.5 point for each statement for which a true/false response is not known.

i) Despite investigation, no permitted and effective herbicide treatments Points are available, and cutting or mowing alone are not sufficient to eliminate plants of this species.

ii) This species is difficult to control without significant damage to native species because: it is widely dispersed throughout the communities (i.e., does not occur within discrete clumps nor monocultures); it is attached to native species (e.g., vine, epiphytes or parasite); or there is a native plant which is easily mistaken for this invader in: (choose one)

$\$ 50 \%$ of discrete populations of this species; $25 \%$ to $50 \%$ of discrete populations of this species.

iii) Total costs of known control method per acre in first year, including access, personnel, equipment, materials, and re-vegetation if needed are $>\$ 1,500 /$ acre.

Continued on next page 
iv) Further site restoration is necessary following plant death to reverse ecosystem impacts and to restore the original habitat-type (e.g., sand replenishment to alleviate beach erosion after Casuarina equisetifolia are removed) or to prevent immediate re-colonization of the invader.

v) The total area over which management (including survey needs beyond known populations) has been, or would have to be, conducted is: (choose one)

$$
\begin{aligned}
& \$ 500 \text { acres; } \\
& <500 \text { but }>50 \text { acres. }
\end{aligned}
$$

vi) Following the first year of control of this species, it would be expected that sites of former populations would require re-survey or re-treatment, due to recruitment from persistent seeds, spores, or vegetative structures, or by dispersal from outside the site: (choose one)

at least once every year for the next 5 years;

between 1 and 4 times over the next 5 years OR regrowth not known.

vii) Much of the areas to be surveyed and controlled cannot be reached easily by surface vehicle (e.g., ATV, truck, or boat) or cannot easily be treated by an individual carrying a backpack sprayer or hand-held tool.

viii) Occurs in more than 20 discrete populations (at least 1 mile apart) in managed areas (i.e., not cultivated).

ix) The number of viable, independent propagules per mature plant (e.g., seeds, spores, fragments, tubers, etc. detached from parent) is $>200$ per year AND: EITHER they can survive for more than 1 year; OR $>10 \%$ disperse a horizontal distance from the parent plant of at least 10 yards, or 3 times the height of parent plant.

x) Age at first reproduction (by seed or vegetative) is within first $10 \%$ of likely life-span and/or less than 3 months. 
The intention of this section is not to identify attributes that must apply to every high-value species but to find parameters that indicate that the species is of some significant value. Few data are available at the species level on plant production and sales in Florida so it is difficult to develop simple questions for which appropriate information can be found. Sales from chain retail stores may only relate to a few growers but it is likely that these will be high income species because the chain-stores will only select species that they are confident will be popular and sell quickly (especially department and grocery stores).

This section is assessed on the State-wide distribution of the species, NOT by zones.

V Economic Value

1. Does this species have any economic value in Florida?

If NO then write $\mathbf{V}=\mathbf{N o}$ on Response Form, but treat as Low in the conclusions table on page 20. Then Go to Conversion of Index

Scores to Index Categories (page 18)

If YES then Go to question $\mathbf{V}-2$.

2. Is this species sold in national or regional retail stores? (E.g., WalMart, Home Depot, Publix supermarkets.)

If response is YES Then $\mathbf{V}=$ High and Go to Conversion of Index Scores to Index Categories (page 18)

If response is $\mathbf{N O}$ then Go to question $\mathbf{V}-\mathbf{3}$.

3. State-wide are there more than 10 commercial growers of this species? (E.g., search "Plant Locators". For example sources see Other Resources on page 27.)

If response is YES Then $\mathbf{V}=$ High and Go to Conversion of Index Scores to Index Categories (page 18)

If response is $\mathbf{N O}$ then Go to question $\mathbf{V}$ - 4 .

4. Does this species have economic value for forage, biomass, or remediation purposes?

If net value $\$ \$ 50,000$ / yr then $\mathbf{V}=$ High and Go to Conversion of Index Scores to Index Categories (page 18)

If net value $<\$ 50,000 /$ yr then $\mathbf{V}=$ Low and Go to Conversion of Index Scores to Index Categories (page 18) 


\section{Conversion of Index Scores to Index Categories}

The actual scores for each index will be important if species are to be compared with each other or over time. For the purposes of reporting the status of a species for each index and for determining the appropriate conclusion, the index scores are converted to index categories. For Potential for Expansion, Management Difficulty, and Economic Value there are only two categories (Low or High). For Ecological Impacts there are four categories Low, Medium, High and Very High.

The criteria for the Impact score cut-offs for each category are based on the combinations of statements that are scored in Section II-a on pages 9 - 10. Low impacts include only one midscoring statement ( 8 points) or one to three low scoring statements ( 1 or 4 points), usually without a wide habitat range. Medium impacts have at least one high scoring statement (12 or 15 points), a mid- and low-scoring statement, multiple low-scoring statements, or a wide habitat range. Most High impacts include an ecosystem effect and/or wide habitat range, plus at least one of the other high-scoring statements or several other low-scoring statements. Very High impacts must show ecosystem effects and either all other impacts or a wide habitat range, or must have all impacts other than ecosystem and must have a wide habitat range.

\section{Conversion of Index Scores to Index Categories}

Using the following table, determine the appropriate category (Low to High or Very High) for each index. (Categories for Potential for Expansion Index and Economic Value were determined on pages 12,13 , and 17)

$\begin{array}{lccc}\text { Category } & & \text { Impacts } & \\ \text { Low } & \text { (L) } & <12 & <15 \\ \text { Medium } & \text { (M) } & 12-26.4 & \\ \text { High } & \text { (H) } & 26.5-41 & \$ 15 \\ \text { Very High } & \text { (VH) } & >41 & \end{array}$

NOTE: It is important to note that the following conclusions would be subject to any local laws, rules, or ordinances that prohibit the sale, transportation, or use of particular species. This may apply to species that are highly problematic in some zone(s), but that have caused minimal impact and have low potential for spread into another zone. 


\section{Conclusions For Zones where conclusions were NOT already developed in Section D}

Conclusions are derived separately for each zone from the combined index categories using the table on page 20. Whenever new information becomes available about the invasive status of a species (e.g., new populations, new data on ecological impacts) that species should be reviewed and if necessary reassessed. The following text corresponds to the abbreviations in the table on page 20 (text in bold is approved language for IFAS documents, text in parentheses provides additional instructions to IFAS faculty and for reassessment):

$O K=$

Not considered a problem species at this time (may be recommended by IFAS faculty and reassess in 10 years).

\section{Caution $=$}

Caution - manage to prevent escape (may be recommended by IFAS faculty and reassess in 2 years).

No - unless limited use approved $=$

This species must be evaluated via Section D.

If the response to Section D is NO or if a proposal for specified and limited use has not been approved by the IFAS Invasive Plant Working Group (IPWG) the conclusion is:

Invasive and not recommended by IFAS faculty (reassess in 10 years - a proposal for specified and limited use may be submitted to the IPWG at any time). In IFAS publications, reference can be made to the web site for the Center for Aquatic and Invasive Plants (http://plants.ifas.ufl.edu/) to determine if any specified and limited uses have been approved since the time of publication.

If the response to Section D is YES and a proposal for specified and limited use has been approved by the IPWG the conclusion is:

\section{Invasive and not recommended by IFAS faculty except for "the specified and limited" use that has been approved by the IFAS Invasive Plants Working Group (reassess in 2 years).}

In IFAS publications the term "the specified and limited" would be replaced by a summary of the specific use that has been approved (e.g., indoor foliage). Details of approved specified and limited uses are to be kept with other assessment documentation.

No $=$

Invasive and not recommended by IFAS faculty (reassess in 10 years). 
Index Categories

$\mathrm{L} / \mathrm{H}=$ either Low or High

Impact Potential Manage. Value

$\mathrm{VH} \quad \mathrm{L} / \mathrm{H} \quad \mathrm{L} / \mathrm{H} \quad \mathrm{L} / \mathrm{H} \quad *$

$\begin{array}{lll}\mathrm{H} & \mathrm{H} & \mathrm{H} \\ \mathrm{H} & \mathrm{H} & \mathrm{H} \\ \mathrm{H} & \mathrm{H} & \mathrm{L} \\ \mathrm{H} & \mathrm{H} & \mathrm{L} \\ \mathrm{H} & \mathrm{L} & \mathrm{H} \\ \mathrm{H} & \mathrm{L} & \mathrm{H} \\ \mathrm{H} & \mathrm{L} & \mathrm{L} \\ \mathrm{H} & \mathrm{L} & \mathrm{L}\end{array}$

$\mathrm{M} \quad \mathrm{H} \quad \mathrm{H}$

$\begin{array}{lll}M & \mathrm{H} & \mathrm{H}\end{array}$

$\begin{array}{lll}M & \mathrm{H} & \mathrm{L}\end{array}$

$\begin{array}{lll}M & \mathrm{H} & \mathrm{L}\end{array}$

M L $\quad \mathrm{H}$

M L $\quad \mathrm{H}$

M L $\quad$ L

M

L $\quad \mathrm{H} \quad \mathrm{H}$

L $\quad H \quad H$

L H

L $\quad \mathrm{H} \quad \mathrm{L}$

L L
$\mathrm{H}$

$\mathrm{H}$

L

L

$\mathrm{H}$

$\mathrm{H}$

L

$\mathrm{L}$

H

L

(n)

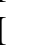

$\mathrm{L}$

L

$\mathrm{H}$

$\mathrm{H}$

$\mathrm{L} / \mathrm{H} \quad \mathrm{L} / \mathrm{H}$
L

\section{Conclusions}

See page 19 for full text for conclusions

No

No
unless

Caution OK

limited use

approved

$*$

$*$

$*$

$*$

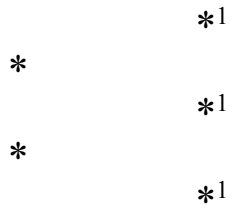

$*$

$*$

$*$

$* 1$

$* 1$

$* 1$

$* 1$

Footnotes:

${ }^{1}$ Go To Section D (page 24) to determine whether this species qualifies for a conclusion of Invasive and not recommended by IFAS faculty except for the specified and limited use that has been approved by the IFAS Invasive Plants Working Group.

${ }^{2}$ If a zone is invaded and has Impact $=$ Low or Medium but the adjacent zone has Impact $=$ High or Very High or has received an Invasive and not recommended by IFAS faculty OR Invasive and not recommended by IFAS faculty except for the specified and limited use that has been approved by the IFAS Invasive Plants Working Group conclusion via Section C, then for the invaded zone under consideration Go To Section C (page 23).

${ }^{3}$ For zones where a species has not invaded, if Potential $=$ Low but Impacts in an adjacent invaded zone are Medium, High, or Very High, then use Caution - manage to prevent escape for the un-invaded zone. If Impact = Low in the adjacent zone or it is not yet invaded, then retain the conclusion Not considered a problem species at this time. 
Section A (from Section I-a page 6 or Section I-b page 8)

The purpose of this section is to identify species that pose a threat of genetic invasion of Federal- or Florida-listed plants or economically-important species, and to identify for further assessment species that have either been introduced recently or that may have the potential to cause problems based on experiences in similar regions of the world. Plants that do not fall into any of these categories are not considered invasive at this time but should be reassessed every 10 years or if invasion into natural areas is recorded.

Documentation of evidence or provision of distributional evidence (as defined in the glossary) must be provided for a YES answer.

A1 Does this species hybridize with any Federal or Florida-listed Species of Special Concern, Threatened or Endangered plants or economically-important species? (E.g., exhibit pollen / genetic invasion.)

If YES then Go to Section D (page 24) If NO then Go to question A2

A2 Has this species been introduced to Florida within the last 10 years if herbaceous, or last 20 years if woody?

If YES then Use a predictive tool If NO then Go to question A3

A3 Does this species have a record of causing problems in other regions with similar habitats and climate to Florida?

If YES then Use a predictive tool

If NO then Not considered a problem species at this time and may be recommended by IFAS faculty but reassess if invasion of natural areas is recorded or within 10 years, whichever is earlier. 
Section B (from Section II-c page 11)

The purpose of this section is to determine whether the locations, in which impacts of the invading species are high, can be clearly defined. If impacts are restricted to an identifiable habitat or community, the species may not be recommended for use near those areas. Dispersal characteristics are evaluated to determine how far away a no-plant zone would have to extend to reasonably ensure that propagules would not reach a site that is susceptible to damaging invasion.

Documentation of evidence (as defined in the glossary) must be provided for YES answers in this section.

B1 Can the habitats and communities in which the worst-case ecological impacts occur (statements i to $\mathrm{v}$ in Section II-a) be clearly defined as different from invaded sites where there are no such impacts (e.g., defined by edaphic or biological factors)? (If ecological impacts include negative effects on a Federal or Florida-listed Species of Special Concern, Threatened or Endangered species, then the specific habitats in which those protected species occurs must be clearly distinguishable from habitats in which they do not occur.)

If NO then return to Section III (page 12)

If YES prepare such a site definition, then Go to question B2

B2 Can an estimate be made of the maximum distance that propagules (or pollen if hybridization is a concern) might reasonably be expected to disperse?

If NO then return to Section III (page 12)

If YES then define that distance of dispersal, complete Sections III, IV, and

V (page 12 - 17) to derive information on Potential for Expansion, Management Difficulty and Economic Value, then Go to Section D (page 24) 
Section C (from Footnote 2 on page 20)

For a zone that is invaded and has Impact $=$ Low but where the adjacent zone has Impact $=$ High or Very High, this section addresses the concern that some of these species have just arrived in a zone and so have not yet become severe enough to be causing ecological impacts in that zone. However, if the species had not yet been detected in this zone, but was considered likely to survive and cause impacts there, the conclusion would match that for the nearest invaded zone (via Section III-c). For example, in 2001, Paederia foetida in the south zone fit this category as a single, new population was first recorded in 2000.

This not only seems inconsistent but risks under-estimating the threat posed by a species at the earliest stage of invasion, when the most effective control action might be taken. On the other hand, if a species has been known in a zone for a long time but it still causes minimal ecological impacts (e.g., Lantana camara in the north zone) a Caution - manage to prevent escape OR Not considered a problem species at this time conclusion would be appropriate.

Documentation of evidence or provision of distributional evidence (as defined in the glossary) must be provided for a YES answer.

C1 Was the first record of this species in natural areas of this zone less than 10 years ago if herbaceous or less than 20 years ago if woody?

If YES then Go to question C2

If NO then the conclusion for this zone remains as Caution - manage to prevent escape OR Not considered a problem species at this time.

C2 Can this species reproduce and disperse in this zone?

If YES then the conclusion for this zone is Invasive and not recommended by IFAS faculty OR Invasive and not recommended by IFAS faculty except for the specified and limited use that has been approved by the IFAS Invasive Plants Working Group to match the adjacent, highly impacted zone. However, do not alter the Ecological Impact category for this zone from Low or Medium. (Thus, if there is an adjacent non-invaded zone, the Ecological Impact category for that zone will remain Low or Medium.) The assessment for this zone can be considered complete now, even if the "documentation of evidence" requirement for Ecological Impacts is not fulfilled (i.e., there are only one or two expert opinions on this species in this zone).

If NO then the conclusion for this zone remains as Caution - manage to prevent escape OR Not considered a problem species at this time 
Section D (from Section A1, Section B, or Footnote 1 on page 20)

This section provides the possibility of a specified exemption to certain Invasive and not recommended by IFAS faculty conclusions (listed above), based on a reasonable certainty that plants grown in the specified conditions will not be able to escape into natural habitats or into the well-defined habitats in which limited impacts occur. Anyone using this assessment tool should recognize that the proposed conclusions have been developed under the assumption that growers, retailers, and consumers will follow any use-specifications made.

D1 Are there specific circumstances in which this species could be used that would not be expected to result in escape and invasion (e.g., foliage plants that are only used indoors and which can be reasonably prevented, by conspicuous labeling, from use or disposal in the landscape) OR is it possible to define how to avoid dispersal of this species to habitats where its impacts are high (i.e., from Section B)?

If NO, then complete the rest of the assessment to derive a conclusion on page 20 , unless this section was reached from Section A1 or Footnote 1 on page 20 in which case the conclusion is Invasive and not recommended by IFAS faculty.

Reassess this species in 10 years.

If YES, then based on a proposal that is approved by the IPWG the conclusion becomes Invasive and not recommended by IFAS faculty except for "the specified and limited" use that has been approved by the IFAS Invasive Plants Working Group. The proposal for specified and limited use should document how invasion would be prevented, and should stipulate that disposal of any propagules must ensure their destruction. Reassess this species in 2 years (or in the case of referrals from Section B, immediately if the incidence of worst-case impacts increases above $10 \%$.)

In IFAS publications the term "the specified and limited" would be replaced by a summary of the specific use that has been approved (e.g., indoor foliage). 


\section{Glossary for this assessment:}

Anthropogenic disturbance. Recurring anthropogenic source of soil exposure (e.g., roads, trails, plow lines) and/or vegetation structure change (e.g., mowing) that is not the result of or does not mimic a natural process and that causes partial or total destruction of vegetative biomass. Also includes human-induced changes in natural disturbance regime (e.g., changing the severity of fires; fire suppression in fire-adapted communities or fire in communities not adapted to fire; grazing cattle above the density of native herbivores). In this context, disturbance is likely to facilitate the invasion of rapidly colonizing and persistent species.

Coverage. Visual or quantitative estimate of the relative amount of area in a stratum where the canopy of the non-native species intercepts the light that would otherwise be available for other species in or below that stratum. Estimated cover may be dispersed or continuous in a site. Cover is usually measured when foliage is fully expanded. In the case of species that form a dense, continuous mat of rhizomes or stolons, the percent of the soil surface or upper level occupied by that root mat can be estimated as soil, rather than canopy, cover.

Disturbance. Natural mechanisms that limit biomass by causing its partial or total destruction (e.g., fires caused by lightning; herbivory; flooding; hurricanes).

Discrete populations. A population is a group of individuals of the same species living in the same place and which are much more likely to reproduce with one another than with individuals from another population. For the purposes of this assessment, discrete populations must be at least 1 mile apart. They likely arose by separate, long-distance dispersal events.

Distributional evidence. Search floras, databases, herbaria, etc. (For examples see "Floras, etc." in Other Resources on page 27.) For each relevant record, document: the source (e.g., database, herbarium); the date; the site; the collector; and any other relevant information. If formal documentation is not available, obtain such information (confirmed in writing) from at least two people who have the expertise to identify the particular species.

Documentation of evidence. One publication including relevant, original research will suffice if data are specific to the taxon and zone(s) under evaluation. If such documentation is not available or needs to be up-dated, at least three individuals who have expertise on the particular species and zone in question must be identified. If more than three experts have been identified, use the responses that are most precautionary (i.e., worst assessment of impacts or greatest management needs reported), as consistent with the intent of the assessment. Relevant information (confirmed in writing) must be provided by at least two of the three experts to justify a "yes" response to a question, or to support a score-accruing statement. For example, in Sections II-a and IV, scores are summed for all statements that have each been confirmed either in the literature or by at least two experts. In statement II-a i), if two experts indicate that there have been ecosystem changes 15 points are assigned for this item even if the experts are reporting different types of ecosystem changes. If data in the literature show that a species causes a particular ecological impact but not in the zone under consideration, expert opinion is still needed. However, an expert could give the opinion that this impact is also likely to occur in the zone and that could be one of the three expert opinions needed. Records should be kept of all the experts who were contacted for each species and zone, regardless of whether they provided input. 
Federal- or Florida -listed. Species that are listed by Federal laws or Florida statutes or rules as Threatened, Endangered or Species of Special Concern within the State of Florida. The list of endangered and threatened species of plants is available at: http://www.doacs.state.fl.us/pi/enpp/botany/images/Notes2003.pdf

Also species and habitats of special concern that are regulated by Florida Statute include: mangroves (FL statute 403.9321), sea oats and sea grape (FL statute 370.041), cypress trees (FL statute 590.02). Other types of organism are listed at: http://www.wildflorida.org/species/Endangered-Threatened-Special-Concern-2004.pdf

Invading. A species that forms self-sustaining and expanding populations within a natural plant community with which it had not previously been associated (cf. "invasive" in Vitousek et al. 1995).

Invasive. Invading species that cause documented ecological impacts ( $>$ Low: pages 18 - 20).

Long-term alterations in ecosystem processes. Examples of ecosystem processes that could be altered: erosion and sedimentation rates; land elevation; water channels; water-holding capacity; water-table depth; surface flow patterns; rates of nutrient mineralization or immobilization; soil or water chemistry; and type, frequency, intensity, or duration of disturbance. For further explanation see Gordon (1998).

Native. Species within its natural range or natural zone of dispersal (i.e., within the range it could have, or would have, occupied without direct or indirect introduction and/or care by humans. Excludes species descended from domesticated ancestors) (Vitousek et al. 1995).

Natural areas. Areas of Florida (public or private) with designated management objectives that include the conservation of native biodiversity. While a range of activities may be conducted on these areas (e.g., prescribed fire, low intensity grazing), those activities are designed to be compatible with the conservation objective. State and local governments and some private landowners, for example, manage natural areas both for recreation, grazing, forestry or other harvest values and for conservation values. These areas would be included in the assessment of invasion, with clearly identifiable edges disregarded. Species that invade only adjacent to roads, trails, fire lanes, recent dredge spoil, formerly cultivated areas, expanses of bare soil, etc. should not be identified as invaders with impacts in natural areas because their persistence and spread is only in these clearly anthropogenically disturbed zones in the natural area. Populations of species that spread along these disturbed zones but disperse over 10 yards into more intact natural areas would be included as invading. Ecological impacts would be assessed only where the population has spread into the intact natural areas and not within the source population in the disturbed or formerly cultivated area.

Pollen or genetic invasion. When a native species is displaced by a non-native species through hybridization.

Sites. Locations that can be distinctly described by name (e.g., named State Park or lake) and may be subdivided into distinct habitats and/or communities (e.g., mesic uplands in Acme Park and rocklands in Acme Park). A site may contain more than one discrete population of a species provided that the populations are at least 1 mile apart. 
Stratum. A distinct layer in the architecture of vegetation (e.g., tree canopy, under-story shrubs).

\section{References:}

Gordon, D.R. 1998. Effects of invasive, non-indigenous plant species on ecosystem processes: lessons from Florida. Ecological Applications 8: 975-989.

Vitousek, P., L. Loope, C. D’Antonio and S.J. Hassol. 1995. Biological invasions as global change. pp. 213-336 In: S.J. Hassol and J. Katzenberger (eds) Elements of change 1994. Aspen Global Change Institute, Aspen, CO.

\section{Other Resources:}

Floras, databases, herbaria, etc.

Search FL Department of Environmental Protection / FLEPPC Exotic plant database http://www.fleppc.org/database/data intro.htm

Atlas of Florida Vascular Plants - Institute for Systematic Botany, University of South

Florida. http://www.plantatlas.usf.edu/ - use scientific name search.

\section{Virtual herbarium - Fairchild Tropical Gardens}

http://www.virtualherbarium.org/ (Information and instructions for using search engine).

To search FTG herbarium directly - http://www.virtualherbarium.org/vh/db/main.htm

Floristic Inventory of South Florida - Institute for Regional Conservation

http://www.regionalconservation.org/ircs/index.cfm

To search IRC database (must register and acquire password)

http://www.regionalconservation.org/ircs/DatabaseChoice.cfm

Information may also be obtained by visiting the University of Florida (FLAS) Herbarium Details regarding herbarium visits may be obtained at:

http://www.flmnh.ufl.edu/natsci/herbarium/flasvasc.htm

Some FLAS herbarium records are available on-line and can be linked through the homepage.

\section{Plant locators.}

Florida Nursery, Growers and Landscape Association http://www.fnga.org/

Search for wholesale commercial availability of a species at:

http://www.fnga.org/locator/searchPlants.asp

\section{Tampa Bay Wholesale Growers Association}

Search for wholesale commercial availability of a species (86 nurseries) at: http://www.tbwg.org/search.cfm

\section{PLANTFINDER - Betrock's Hortworld Web site}

Search for wholesale commercial availability of a species in Florida at: http://www.plantfinder.com/availability/plantavailability1.asp 